\title{
Selective inhibition of JNK mitochondrial location is protective against seawater inhalation-induced ALI/ARDS
}

\author{
LIYAN BO ${ }^{1-3^{*}}$, YANYAN $\mathrm{LI}^{2 *}$, WEI LIU ${ }^{2}$, FAGUANG JIN ${ }^{2}$ and CONGCONG LI ${ }^{1}$ \\ ${ }^{1}$ Department of Respiratory and Critical Care Medicine, General Hospital of Northern Theater Command, \\ Shenyang, Liaoning 110016; ${ }^{2}$ Department of Respiratory and Critical Care Medicine, Tangdu Hospital, \\ Fourth Military Medical University; ${ }^{3}$ Department of Respiratory and Critical Care \\ Medicine, Chest Hospital of Xi'an, Xi'an, Shaanxi 710038, P.R. China
}

Received November 13, 2020; Accepted April 21, 2021

DOI: $10.3892 / \mathrm{mmr} .2021 .12154$

\begin{abstract}
Localization of phosphorylated (p)-JNK to the mitochondria can lead to functional mitochondrial disorder, resulting in a decrease in energy supply and membrane potential, as well as an increase in reactive oxygen species production and apoptosis. JNK is involved in the occurrence of acute lung injury (ALI), and activation of the JNK pathway is one of the crucial factors resulting in injury. The aim of the present study was to investigate whether the JNK-mitochondria (mitoJNK) location participated in the occurrence of ALI and acute respiratory distress syndrome (ALI/ARDS). The present study examined the activation of the JNK pathway, the content of JNK located on the mitochondria and the treatment effects of a cell-permeable peptide Tat-Sab ${ }_{\text {KIM1 }}$, which can selectively inhibit the location of JNK on mitochondria. The expression levels of proteins were detected by western blot analysis. Lung injuries were evaluated by histological examination, wet-to-dry weight ratios, and $\mathrm{H}_{2} \mathrm{O}_{2}$ and malondialdehyde concentrations in the lung tissues. Lung cells apoptosis was evaluated using TUNEL assay. The results demonstrated that JNK was phosphorylated and activated during seawater inhalation-induced ALI/ARDS, not only in the routine JNK pathway but also in the mitoJNK pathway. It was also found that Tat-Sab ${ }_{\mathrm{KIM} 1}$ could specifically inhibit JNK localization to mitochondria and the activation of mitoJNK signaling. Furthermore, Tat-Sab ${ }_{\text {KIMI }}$ could inhibit Bcl-2-regulated autophagy and mitochondria-mediated apoptosis. In conclusion, mitoJNK localization disrupted the
\end{abstract}

Correspondence to: Dr Congcong Li, Department of Respiratory and Critical Care Medicine, General Hospital of Northern Theater Command, 83 Wenhua Road, Shenhe, Shenyang, Liaoning 110016, P.R. China

E-mail: licong1988@hotmail.com

*Contributed equally

Key words: JNK, acute lung injury and acute respiratory distress syndrome, mitochondria, apoptosis, autophagy normal physiological functions of the mitochondria during ALI/ARDS, and selective inhibition of JNK and mitochondrial SH3BP5 (also known as Sab) binding with Tat-Sab ${ }_{\mathrm{KIM} 1}$ can block deterioration from ALI/ARDS.

\section{Introduction}

JNK belongs to the family of MAPKs and encompasses three encoded genes, JNK1, JNK2 and JNK3 (1). JNK1 and JNK2 are widely expressed in various tissues, whereas $J N K 3$ is primarily expressed in the brain and heart $(1,2)$. JNK is also known as stress-activated kinase, which is involved in the regulation of the response of the body to external or internal stimuli, inflammation and cell apoptosis (3). As a response to a specific stimulation, MAPKs [such as MAPK kinase (MKK) 4 and MKK7] activate JNK via phosphorylation (4), and subsequently, regulate the phosphorylation and activity of several downstream factors, such as activating transcription factor-2 (5), ETS transcription factor (6) and nuclear factor of activated T-cells (7). Among these factors, c-jun/activator protein-1 (AP-1) has been clearly elucidated with respect to the JNK regulatory pathway. JNK can activate AP-1 via phosphorylation, followed by the regulation of cell proliferation and apoptosis (8). Several pathological studies $(9,10)$ have reported the abnormal activation of the JNK signaling pathway and its involvement in the modulation of cell apoptosis, and inhibition of this pathway can reduce the proportion of apoptotic cells under pathological conditions (9). Furthermore, JNK can be activated via phosphorylation, and bind to the outer membrane mitochondrial protein mitochondrial SH3BP5 (also known as $\mathrm{Sab}$ ), thereby regulating the function of this organelle (11). The localization of p-JNK to mitochondria can lead to its functional disorder, resulting in a decrease in the energy supply and membrane potential, as well as an increase in reactive oxygen species (ROS) production and the occurrence of apoptosis $(8,9,12)$.

Previous studies (13-15) have shown that JNK was involved in the occurrence of acute lung injury (ALI) and served an important role in the regulation of apoptosis in lung tissue. Experimental studies have revealed that the activation of the JNK pathway was one of the crucial factors resulting in injury; this pathway also interacts with the NF- $\mathrm{KB}$ 
pathway, intercellular tight junction proteins and TGF- $\beta$ to participate in the development of lung injury $(13,16)$. The pathological changes associated with ALI are partly due to the abnormal regulation of mitochondria, such as the production of ROS, induced autophagy and apoptosis (17-19). Moreover, maintaining the stability of mitochondrial function is vital to ameliorate ALI/acute respiratory distress syndrome (ARDS) (20,21). Mitochondria, as intracellular energy sources, serve a crucial role in cell survival and the normal physiological function of organelles, and maintaining mitochondrial stability is essential for cells, tissues and organs (22). Activation of the JNK pathway has been reported to disrupt the function of mitochondria in myocardial ischemia-reperfusion injury, and as a result, decreases the function and survival of myocardial cells (8). However, activation of JNK-mediated mitochondrial function abnormalities is rarely reported with respect to the occurrence and progression of ALI/ARDS. In the present study, we hypothesized that abnormal activation of the JNK-mitochondrial (mitoJNK) pathway could significantly disrupt the normal physiological function of lung cells, resulting in the occurrence of ALI/ARDS. Moreover, the underlying role of the mitoJNK pathway in ALI remains unknown, and requires further study.

\section{Materials and methods}

Reagents. Antibodies (Ab) against cytochrome c (cat. no. ab133504), Bcl-2 (cat. no. ab196495), cytochrome c oxidase IV (COX IV) (cat. no. ab202554) and GAPDH (cat. no. ab181602) were purchased from Abcam. Phosphorylated (p)-Bcl-2 (Ser70) rabbit monoclonal (m)Ab (cat. no. \#2827), p-JNK (Thr183/Tyr185) rabbit mAb (cat. no. \#4668) and JNK rabbit mAb (cat. no. \#9258) were obtained from Cell Signaling Technology, Inc. LC3 rabbit mAb (cat. no. sc-398822) was purchased from Santa Cruz Biotechnology, Inc. The malondialdehyde (MDA) Assay kit (TBA method) (cat.no. A003-1) and $\mathrm{H}_{2} \mathrm{O}_{2}$ Assay kit (cat. no. A064-1) were obtained from Nanjing Jiancheng Bioengineering Institute. An In Situ Cell Death Detection kit was obtained from Roche Diagnostics $\mathrm{GmbH}$. Dexamethasone (DXM) was purchased from Sigma-Aldrich (Merck KGaA). Tat-Sab ${ }_{\text {KIM1 }}$ (GFESLSVPSPLDLSGPRVVAP PRRRQRRKKRG-NH ${ }_{2}$ ) was purchased from NeoPeptide. Seawater (osmolality 1,300 mmol/1; pH 8.2; $26.518 \mathrm{~g} / \mathrm{l} \mathrm{NaCl}$; $3.305 \mathrm{~g} / 1 \mathrm{MgSO}_{4} ; 2.447 \mathrm{~g} / 1 \mathrm{MgCl}_{2} ; 1.141 \mathrm{~g} / 1 \mathrm{CaCl}_{2} ; 0.725 \mathrm{~g} / \mathrm{l}$ $\mathrm{KCl} ; 0.202 \mathrm{~g} / 1 \mathrm{NaHCO}_{3} ; 0.083 \mathrm{~g} / 1 \mathrm{NaBr}$ ) was prepared according to the major composition of the East China Sea provided by the Chinese Ocean Bureau (23).

Animal procedures. The animal procedures in this study were approved by the Animal Care and Use Committee of The Fourth Military Medical University (approval no. TDLL20160193), and were carried out in accordance with the Declaration of the National Institutes of Health Guide for Care and Use of Laboratory Animals (24). A total of 32 male Sprague-Dawley (SD) rats (weight, 180-220 g; age, 6 weeks) were maintained on a light/dark cycle of 12:12-h with free access to food and water. The rats were maintained in an atmosphere with an ambient temperature of $18-26^{\circ} \mathrm{C}$ and relative humidity of $40-70 \%$. The SD rats were randomly divided into the following experimental groups $(n=8)$ : i) Control group; ii) seawater inhalation groups; iii) DXM pre-treatment group, in which the rats were pre-treated intraperitoneally with DXM $(2.5 \mathrm{mg} / \mathrm{kg}$ body weight) 30 min before modelling; and iv) Tat-Sab ${ }_{\text {KIM1 }}$ pre-treatment group, in which the rats were pre-treated with Tat-Sab ${ }_{\text {KIM1 }}(2 \mathrm{mg} / \mathrm{kg}$ body weight $)$ via tracheal injection $10 \mathrm{~min}$ before modelling.

Seawater inhalation-induced ALI/ARDS was established through the following procedures. First, the rats were anesthetized with sodium pentobarbital (45 $\mathrm{mg} / \mathrm{kg}$ weight) intraperitoneally. Then, the animals were placed in the supine position with the head elevated at an angle of $30^{\circ}$ during the experiments. A 1-ml syringe was gently inserted into the trachea at $\sim 1.5 \mathrm{~cm}$ above the carina. Subsequently, $4 \mathrm{ml} / \mathrm{kg}$ body weight seawater was instilled into both lungs within $4 \mathrm{~min}$. The lungs were then harvested after the rats were sacrificed via intraperitoneal injection of $200 \mathrm{mg} / \mathrm{kg}$ sodium pentobarbital at the predetermined time $(6 \mathrm{~h})$.

Western blot analysis. The protein extract prepared from the lung tissue harvested $6 \mathrm{~h}$ after seawater instillation was subjected to western blot analysis, as described previously (25). Briefly, the protein was obtained using RIPA lysis buffer (Beyotime Institute of Biotechnology) and protein concentration was quantified using a Bradford kit (Beyotime Institute of Biotechnology) according to the manufacturer's instructions. Total proteins $(20 \mu \mathrm{g})$ were separated by SDS-PAGE on $12 \%$ gels and transferred to nitrocellulose membranes (EMD Millipore). Then, the membranes were blocked with $10 \%$ non-fat dry milk in TBS at room temperature for $30 \mathrm{~min}$ and probed at $4{ }^{\circ} \mathrm{C}$ overnight with primary Abs, including anti-p-JNK (1:1,000), anti-JNK (1:1,000), anti-GAPDH $(1: 2,500)$, anti-COX IV (1:1,000), anti-LC3 (1:1,000), anti-p-Bcl-2 (1:1,000), anti-Bcl-2 (1:1,000) or anti-cytochrome c $(1: 10,000)$. Subsequently, the membranes were washed with TBS-0.3\%Tween-20 and then incubated with an appropriate HRP-conjugated secondary Ab $(1: 10,000)$ at room temperature for $2 \mathrm{~h}$. The immunoreactive target proteins were detected using an ECL detection system (Thermo Fisher Scientific, Inc.). Band intensities were semi-quantified using Image Lab 4.1 (Bio-Rad Laboratories, Inc.).

Histopathological evaluation. The lung tissue was harvested and fixed with $4 \%$ paraformaldehyde at room temperature for $24 \mathrm{~h}$, embedded in paraffin and cut into $5-\mu \mathrm{m}$ sections that were stained with $H \& E$ at room temperature for $3 \mathrm{~min}$. Sections were examined with an optical microscope (magnification, x100).

Preparation of mitochondrial and cytosolic/nuclear proteins. Mitochondrial and cytosolic/nuclear proteins were prepared by isolating mitochondria from the cells, as described previously (9). Briefly, isolation buffer $(210 \mathrm{mM}$ mannitol; $70 \mathrm{mM}$ sucrose; 5 mM HEPES; 1 mM EGTA; 0.5 mg/ml BSA (Merck $\mathrm{KGaA}$; $\mathrm{pH}=7.4$ ) was used to wash and homogenize the rat lungs. Then, the homogenate was centrifuged at $1,000 \mathrm{x} \mathrm{g}$ for $10 \mathrm{~min}$ at $4^{\circ} \mathrm{C}$. The supernatant was collected and centrifuged at $10,000 \mathrm{x} \mathrm{g}$ for $10 \mathrm{~min}$ at $4^{\circ} \mathrm{C}$. This second supernatant was used as the soluble cytosolic/nuclear fraction with excluded mitochondria, and the sedimentation pellet was resuspended in lysis buffer for western blot analysis of the mitochondrial proteins according to the aforementioned steps. COX IV was 
A

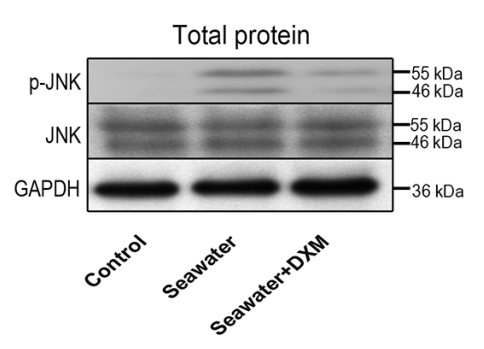

D

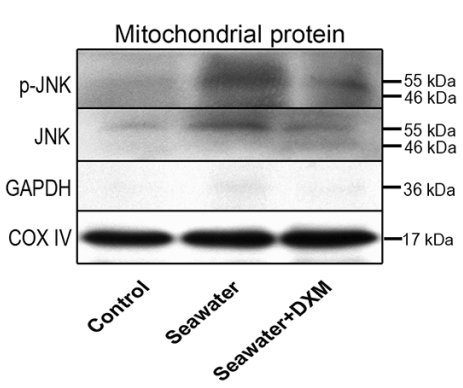

$\mathrm{H}$

H

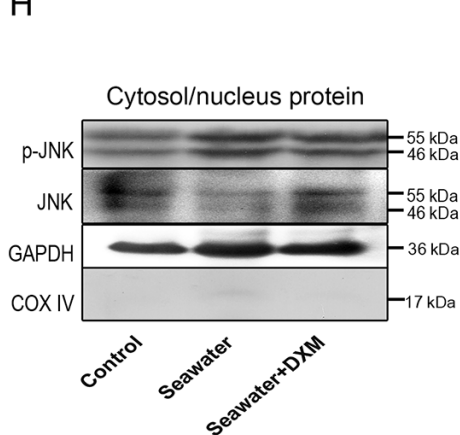

B

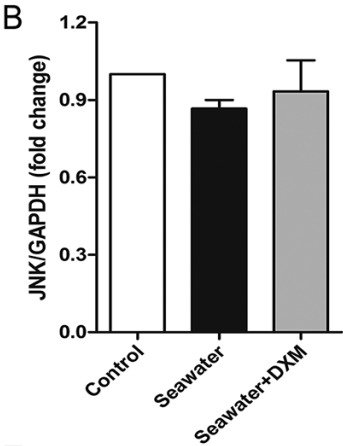

E

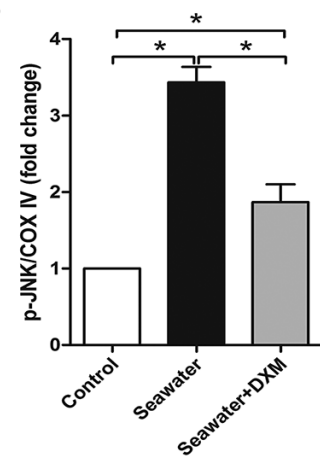

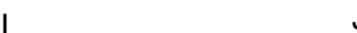

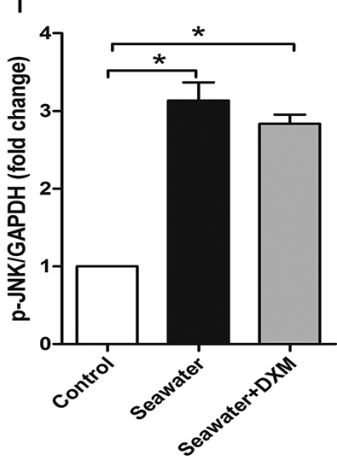

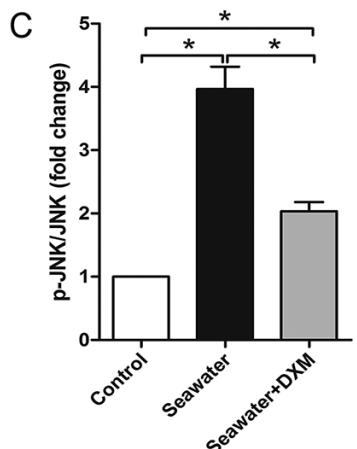

F

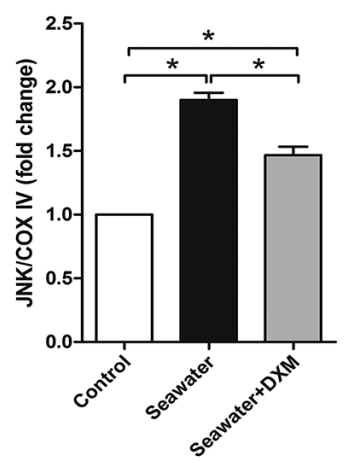

G

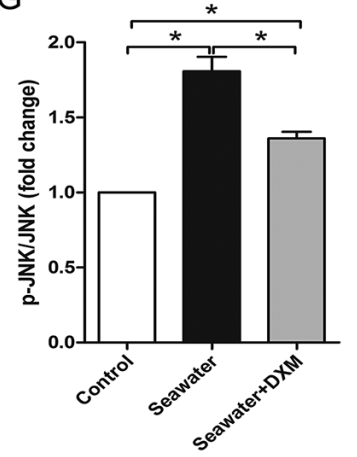

K
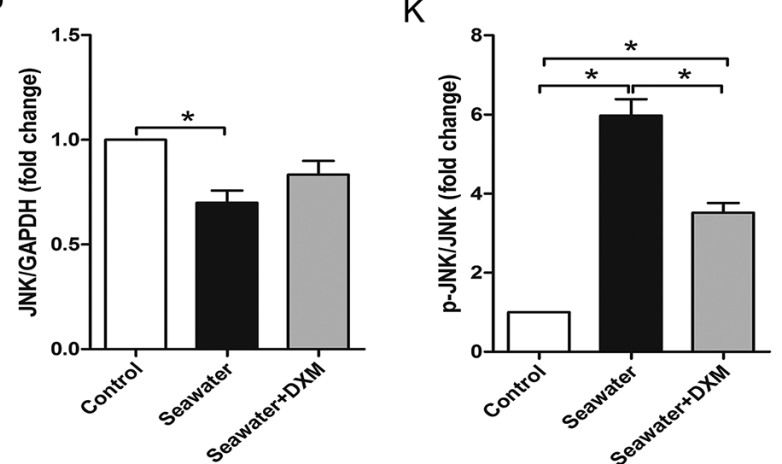

Figure 1. JNK-mitochondria signaling is activated during seawater inhalation-induced acute lung injury/acute respiratory distress syndrome. (A) Representative western blotting images of p-JNK and JNK expression in the total protein. (B) Relative JNK protein content in the total protein. (C) Normalized ratio of p-JNK/JNK of the total protein. (D) Representative western blotting images of p-JNK and JNK expression in the mitochondrial protein. Relative (E) p-JNK and (F) JNK protein content in the mitochondrial protein. (G) Normalized ratio of p-JNK/JNK in the mitochondrial protein. (H) Representative western blotting images of p-JNK and JNK expression in the cytosol/nucleus protein. Relative (I) p-JNK and (J) JNK protein content in the cytosol/nucleus protein. (K) Normalized ratio of $\mathrm{p}-\mathrm{JNK} / \mathrm{JNK}$ of the cytosol/nucleus protein. Data are presented as the mean $\pm \mathrm{SEM}$. $\mathrm{n}=4 / \mathrm{group}$. * $<<0.05$. DXM, dexamethasone; p-, phosphorylated; COX IV, cytochrome c oxidase IV.

used as an internal mitochondrial control, and GAPDH served as the control for other organelles.

Lung wet-to-dry (W/D) weight ratio. The W/D weight ratio of the lung tissue is commonly used to reflect the severity of pulmonary oedema (25). Briefly, the rats were sacrificed at the predetermined time points ( $6 \mathrm{~h}$ after seawater inhalation), and the chests were quickly opened. The same part of the left lung from each rat was weighed as the wet weight after removal of the other tissues. Then, each lung was placed in an oven for baking at $80^{\circ} \mathrm{C}$ for $72 \mathrm{~h}$ to obtain a constant weight, denoted as the dry weight. The W/D weight ratio was obtained by dividing the wet weight by the dry weight of the left lung.

Determination of $\mathrm{H}_{2} \mathrm{O}_{2}$ concentrations and MDA levels. $\mathrm{H}_{2} \mathrm{O}_{2}$ and MDA concentrations in the lung tissues were detected using the $\mathrm{H}_{2} \mathrm{O}_{2}$ Assay kit and MDA Assay kit, respectively, according to the manufacturer's instructions. Briefly, the lung tissue samples were homogenized in cold normal saline (lung tissue to normal saline ratio, 1:9). Then, the homogenate was examined according to the protocol of the kit. To detect the $\mathrm{H}_{2} \mathrm{O}_{2}$ concentration, the rate of change in absorbance was measured with a spectrophotometer at $405 \mathrm{~nm}$. For detecting the MDA concentration, the rate of change in absorbance was measured with a spectrophotometer at $532 \mathrm{~nm}$.

Assessment of lung cells apoptosis. In order to quantify cell apoptosis in the injured rat lungs, a TUNEL assay was conducted using an In Situ Cell Death Detection kit according to the manufacturer's instructions. Briefly, lung tissues were fixed with $4 \%$ paraformaldehyde for $24 \mathrm{~h}$ at room temperature. The lung tissues were then embedded in paraffin and sectioned into $5-\mu \mathrm{m}$ sections. After dewaxing, the tissue sections were incubated with TUNEL working solution for $1 \mathrm{~h}$ at $37^{\circ} \mathrm{C}$ to 
A
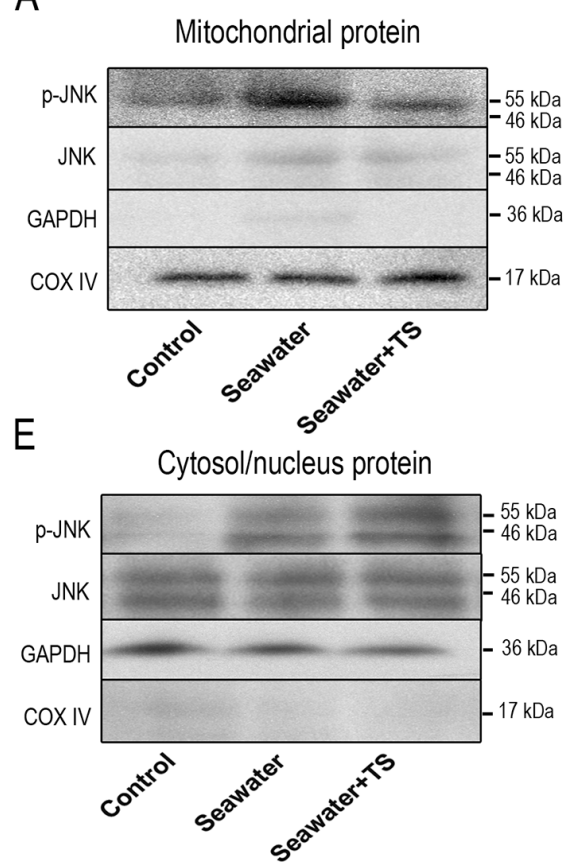

B

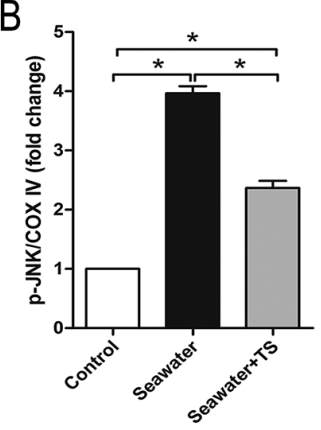

F

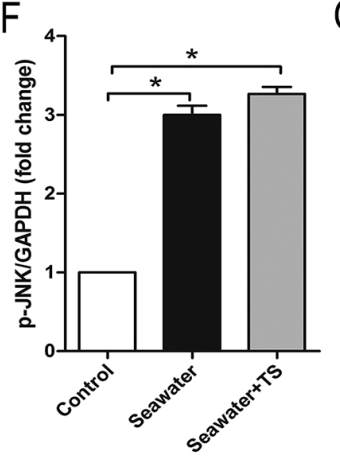

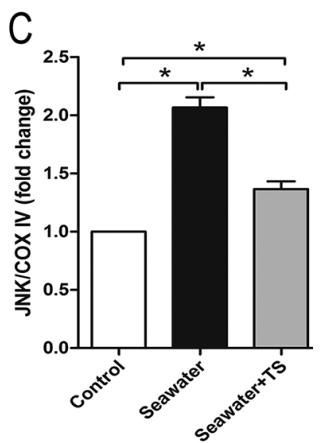

G

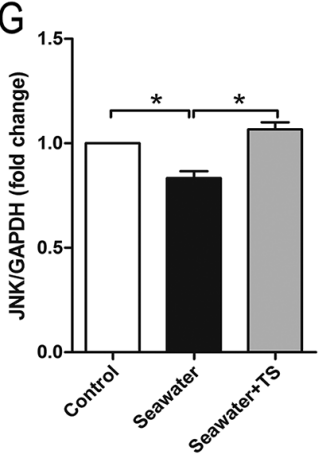

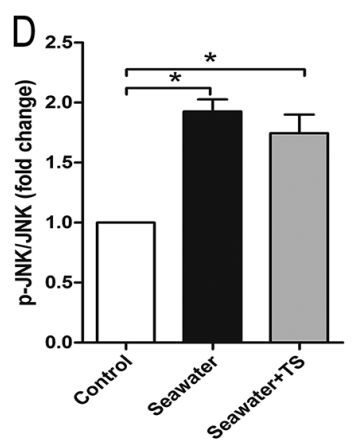

$\mathrm{H}$

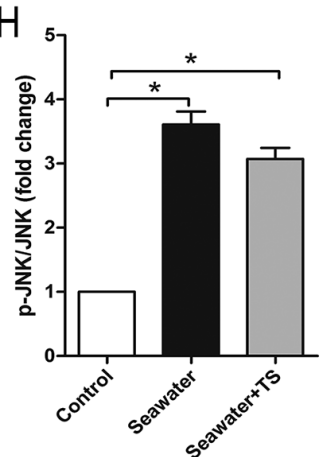

Figure 2. Tat-Sab ${ }_{\text {KIM1 }}$ peptide tracheal injection could inhibit JNK-mitochondrial activation during acute lung injury/acute respiratory distress syndrome. (A) Representative western blotting images of p-JNK and JNK expression in the mitochondrial protein. Relative (B) p-JNK and (C) JNK protein content in the mitochondrial protein. (D) Normalized ratio of p-JNK/JNK in the mitochondrial protein. (E) Representative western blotting images of p-JNK and JNK expression in the cytosol/nucleus protein. Relative (F) p-JNK and (G) JNK protein content in the cytosol/nucleus protein. (H) Normalized ratio of p-JNK/JNK in the cytosol/nucleus protein. Data are presented as the mean \pm SEM. $n=4 /$ group. ${ }^{*} \mathrm{P}<0.05$. TS, Tat-Sab ${ }_{\mathrm{KIM} 1}$; p-, phosphorylated; COX IV, cytochrome c oxidase IV.

label apoptotic cells, and then nuclei were stained with DAPI (Merck KGaA) for $5 \mathrm{~min}$ at room temperature. The slides were mounted with $50 \%$ glycerol (Merck KGaA). Finally, the sections were visualized using a fluorescence microscope (magnification, x200; Olympus Corporation). A total of 10 images from three slides from every group were randomly selected, and the number of cells exhibiting positive staining for apoptosis per field were counted and analysed.

Statistical analysis. Statistical analyses were performed using GraphPad Prism 8 (GraphPad Software, Inc.). The graphical data are presented as the mean \pm SEM and experiments were repeated four times. The experimental groups were compared using a one-way ANOVA and Bonferroni's multiple comparison tests. $\mathrm{P}<0.05$ was considered to indicate a statistically significant difference.

\section{Results}

mitoJNK signaling is activated during seawater inhalationinduced ALI/ARDS. To elucidate the role of JNK, especially mitoJNK, in ALI/ARDS, the current study assessed the expression levels of $\mathrm{p}-\mathrm{JNK}$ and $\mathrm{JNK}$ in the total protein, mitochondrial protein and cytosol/nucleus protein of the lung via western blot analysis. As shown in Fig. 1A, B and C, seawater inhalation significantly phosphorylated JNK, thereby activating the JNK pathway in the lung. Furthermore, it was found that the phosphorylation level of JNK was elevated significantly in both the mitochondria (Fig. 1D, E and G) and cytosol/nucleus (Fig. 1H, I and K) after seawater challenge. In addition, the total JNK content in the mitochondria was also elevated (Fig. 1D and F), whereas that in the cytosol/nucleus (Fig. $1 \mathrm{H}$ and $\mathrm{J}$ ) was decreased, indicating JNK translocation from the cytosol/nucleus to mitochondria during ALI/ARDS.

The protective role of DXM in ALI/ARDS was also investigated. DXM pre-treatment significantly alleviated the high level of phosphorylated JNK (Fig. 1A and C), in both the mitochondria (Fig. 1D and G) and cytosol/nucleus (Fig. $1 \mathrm{H}$ and $\mathrm{K}$ ).

Protective effects of the mitoJNK-inhibiting peptide Tat-Sab ${ }_{\text {KIMI }}$ against ALI/ARDS. To further examine the role of mitoJNK activation in ALI/ARDS, the peptide Tat-Sab ${ }_{\mathrm{KIM} 1}$, which can act on the $\mathrm{Sab}_{\mathrm{KIM} 1}$ domain and be expressed in mitochondria (6), was used. The peptide can also mediate the localization of JNK by selectively blocking JNK translocation to mitochondria both in vitro and in vivo $(12,26)$. However, Tat-Sab ${ }_{\mathrm{KIM} 1}$ does not exhibit any effect on the translocation of JNK to the nucleus (12). Tracheal injection of Tat-Sab ${ }_{\mathrm{KIM} 1} 10 \mathrm{~min}$ before modelling significantly decreased the phosphorylation level of JNK in mitochondria (Fig. 2A and B) and total JNK expression in mitochondria when compared with the seawater inhalation group (Fig. 2A and C); however, it did not influence the ratio of $\mathrm{p}-\mathrm{JNK} / \mathrm{JNK}$ when compared with the seawater inhalation group (Fig. 2D). p-JNK expression in the cytosol/nucleus was elevated in both the seawater inhalation group and Tat-Sab ${ }_{\mathrm{KIM} 1}$ pre-treatment group compared with that in the control group (Fig. 2E and F). The ratio of $\mathrm{p}-\mathrm{JNK} / \mathrm{JNK}$ in the cytosol/nucleus was also elevated after seawater inhalation compared with that in the control group (Fig. 2E and $\mathrm{H}$ ). In addition, as presented in Fig. 2E and G, Tat-Sab ${ }_{\mathrm{KIM} 1}$ increased total JNK expression in the cytosol/nucleus when compared with the seawater 

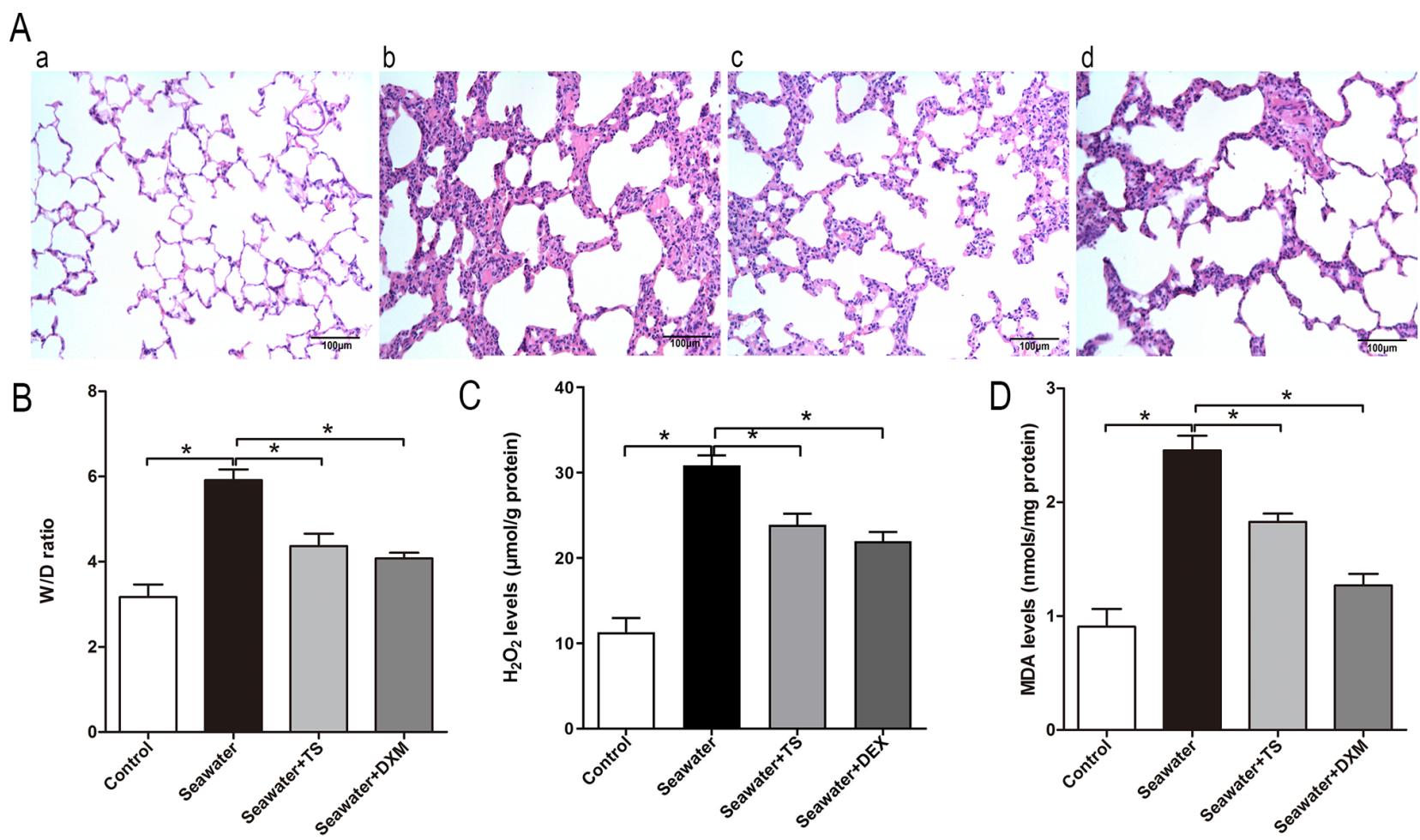

Figure 3. Protective effect of JNK-mitochondrial signaling-inhibiting peptide Tat-Sab ${ }_{\text {KIM1 }}$ against ALI/ARDS. (A) Histological changes in rat lung tissue;

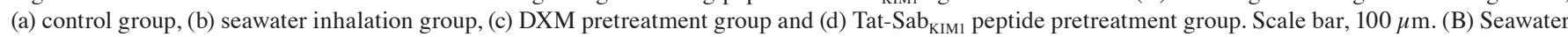
inhalation-induced lung W/D ratio changes. Change of (C) $\mathrm{H}_{2} \mathrm{O}_{2}$ and (D) MDA levels. Data are presented as the mean $\pm \mathrm{SEM}$. $\mathrm{n}=4 / \mathrm{group}$. ${ }^{*} \mathrm{P}<0.05$. $\mathrm{TS}$, Tat-Sab $\mathrm{KIMI}_{1}$; W/D, wet-dry; MDA, malondialdehyde; DXM, dexamethasone.
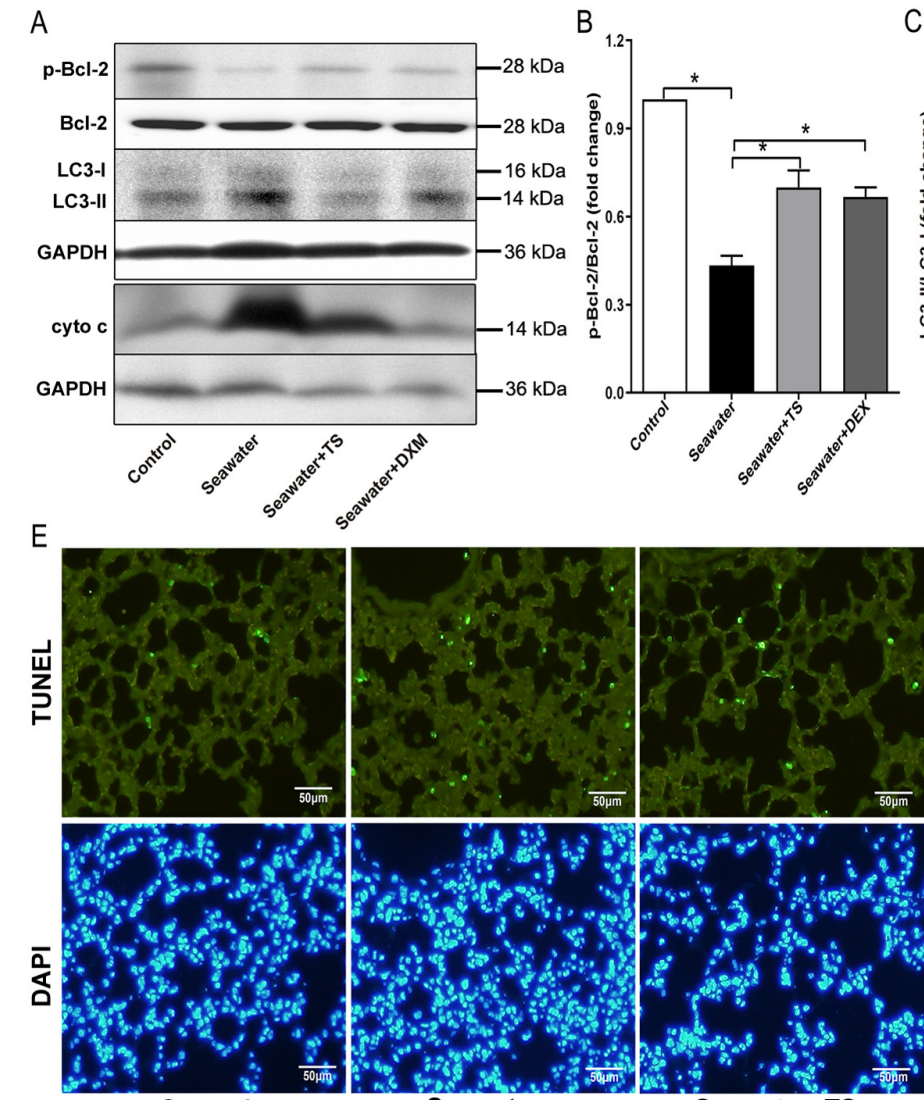

Control

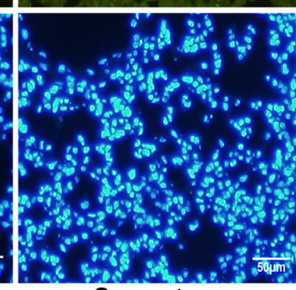

Seawater
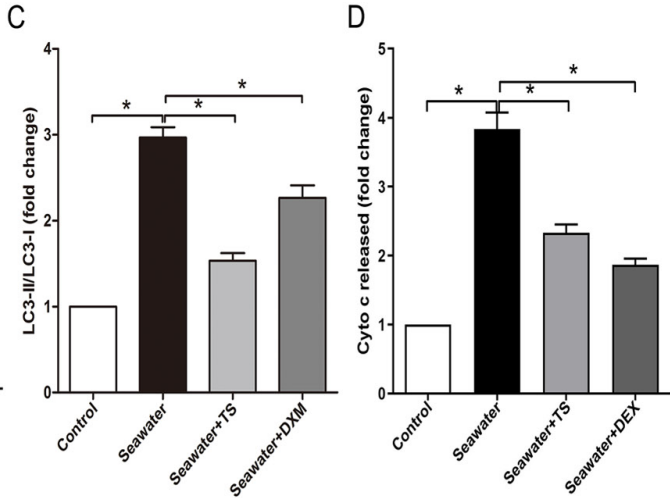

F
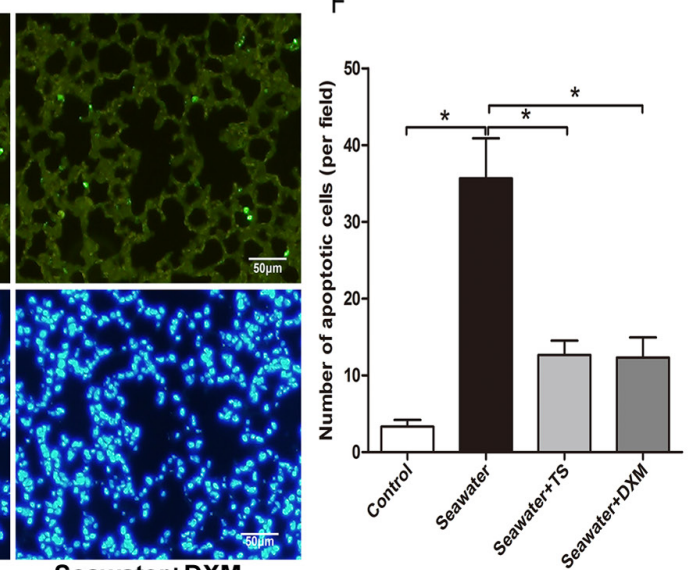

Figure 4. JNK-mitochondrial activation contributes to the occurrence of autophagy and mitochondria-mediated apoptosis. (A) Representative western blotting images of p-Bcl-2, LC3 and cyto c release. (B) Relative p-Bcl-2 protein content. (C) Normalized ratio of LC3-II/LC3-I. (D) Relative content of cyto c released into cytosol. (E) Representative photomicrographs of in situ detection of apoptotic cells using TUNEL staining in lung slices from rats. Scale bar, $50 \mu \mathrm{m}$. (F) Statistical results of apoptotic cells per field. Data are presented as the mean \pm SEM. $n=4 /$ group. ${ }^{*} \mathrm{P}<0.05$. TS, Tat-SabKIM1; cyto c, cytochrome c; DXM, dexamethasone. 


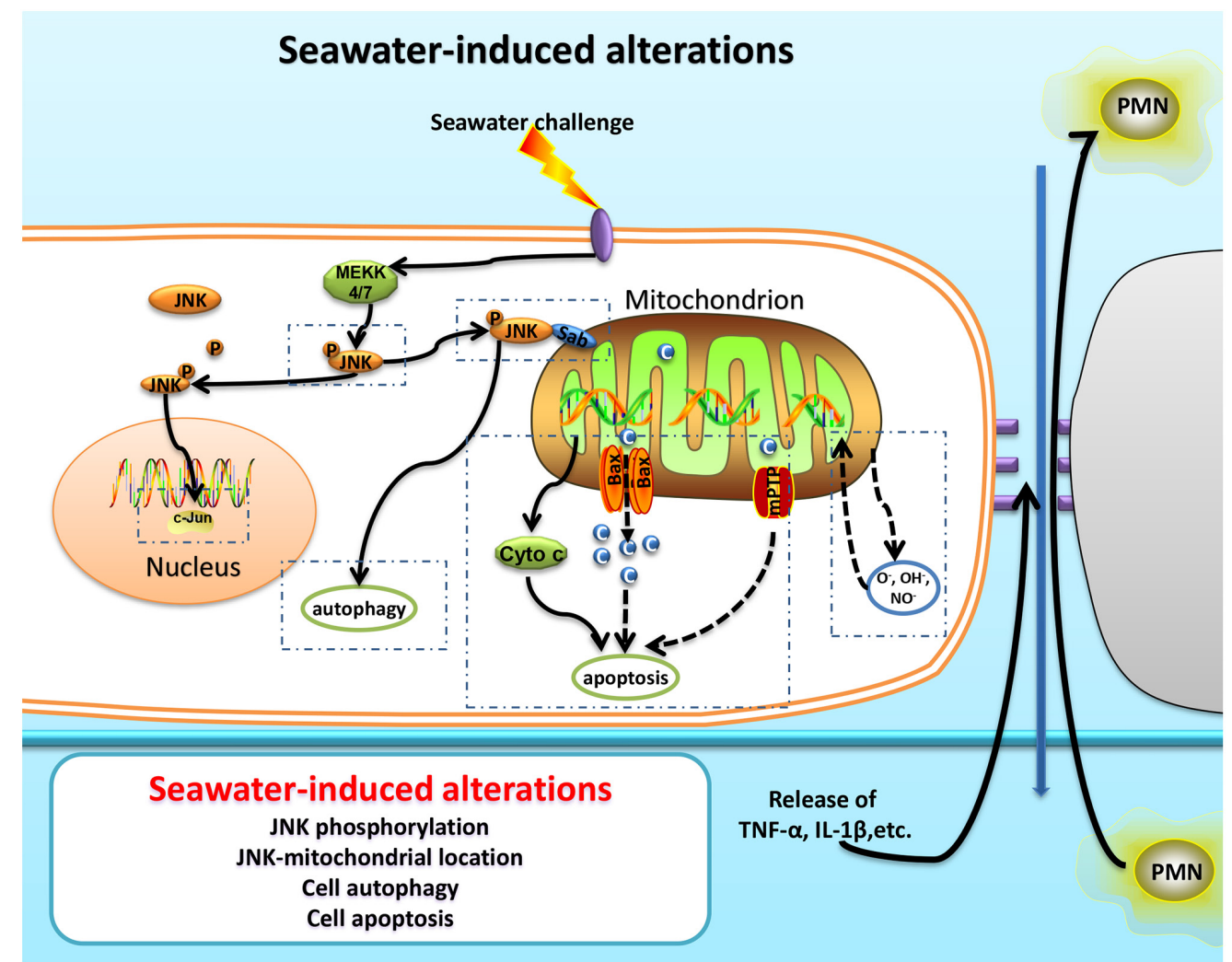

Figure 5. Schematic image depicting the seawater inhalation-induced alterations associated with JNK-mitochondrial activation. PMN, polymorphonuclear cells; C, calcium ion; mPTP, mitochondrial permeability transition pore; Cyto c, cytochrome c; MEKK, MEK kinase; p, phosphorylation.

inhalation group. These results indicated that Tat-Sab $\mathrm{KIM}_{1}$ could specifically inhibit JNK localization to the mitochondria and the activation of mitoJNK signaling, without any impact on the ratio of $\mathrm{p}-\mathrm{JNK} / \mathrm{JNK}$ or the cytosolic/nuclear JNK activation.

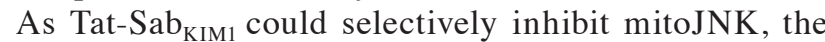
effects of mitoJNK activation on the progression of ALI/ARDS were further evaluated. Tat-Sab $\mathrm{KIM}_{\mathrm{K}}$-mediated inhibition of JNK localization to the mitochondria alleviated the seawater inhalation-induced destruction of lung tissue structure (Fig. 3A) and pulmonary oedema (Fig. 3B). In addition, the inhibitory effects slightly improved the levels of the markers of peroxidation and oxidative stress, $\mathrm{H}_{2} \mathrm{O}_{2}$ and MDA, respectively, compared with the seawater inhalation group (Fig. 3C and D). These results suggested that the inhibition of mitoJNK activation exerted a protective effect against ALI/ARDS. DXM pre-treatment could inhibit JNK phosphorylation in both the mitochondria and cytosol/nucleus compared with that in the seawater group, thus indicating that it did not selectively inhibit mitoJNK (Fig. 1C and G). As shown in Fig. 3A, DXM could also alleviate seawater inhalation-induced lung injury. In addition, the pulmonary oedema and oxidative stress of the DXM pre-treatment group were ameliorated compared with in the seawater inhalation group (Fig. 3B-D).

Effects of mitoJNK activation on the occurrence of autophagy. Autophagy has been reported to participate in the development of ALI/ARDS, as per the conversion of LC3, i.e., LC3-I to LC3-II (27). Bcl-2 may also modulate autophagy via an interaction with the autophagy protein, Beclin 1 (28). Seawater inhalation decreased the expression level of p-Bcl-2 and increased the conversion of LC3 to its active form LC3-II, while the inhibition of mitoJNK by Tat-Sab ${ }_{\mathrm{KIM} 1}$ restored the expression levels of p-Bcl-2 and LC3 (Fig. 4A-C), thereby indicating that the inhibition blocked the autophagy induced by ALI/ARDS.

MitoJNK activation contributes to mitochondria-mediated apoptosis. Next, mitochondria-mediated apoptosis during ALI/ARDS was examined. In the seawater inhalation group, cytochrome c released from mitochondria into the cytosol was significantly increased compared with the control group (Fig. 4A and D). Furthermore, treatment with Tat-Sab $\mathrm{KIM}_{\mathrm{K}}$ or DXM reduced the amount of cytochrome c released from the mitochondria. The findings observed for cell apoptosis, as evaluated via TUNEL staining, were in line with the aforementioned results. The inhibition of mitoJNK signaling had an anti-apoptotic effect (Fig. 4E and F), as Tat-Sab ${ }_{\mathrm{KIM} 1}$ treatment significantly decreased the number of TUNEL-positive cells. These results indicated that mitoJNK signaling participated in mitochondria-mediated apoptosis during ALI/ARDS (Fig. 5).

\section{Discussion}

ARDS is one of the leading causes of morbidity and mortality in critically ill patients, and in the USA, 75,000 individuals die due to ARDS every year (29). Although research and clinical trials have been conducted, the mortality of ARDS continues to be $25-40 \%$, and few pharmacological interventions can improve the mortality rate (30). The pathological changes associated with ALI/ARDS are partly due to the abnormal regulation of mitochondria, including the production 
of ROS, induced autophagy and apoptosis (17-19). Moreover, maintaining the stability of mitochondrial function is vital to ameliorate ALI/ARDS $(20,21)$.

The main aim of the present study was to confirm that the activation of mitoJNK signaling was involved in the development of ALI/ARDS. By inhibiting the interaction of JNK and Sab using a selective peptide, Tat-Sab ${ }_{\mathrm{KIM} 1}$, JNK could not be localized to mitochondria, thereby rendering an opportunity to examine the underlying role of mitoJNK in the development of ALI/ARDS. The present results demonstrated that JNK was phosphorylated and activated during ALI/ARDS, not only in the routine JNK pathway but also in the mitoJNK pathway. Furthermore, the mitoJNK signal was activated during seawater inhalation-induced ALI/ARDS, as shown by JNK translocation from the cytosol/nucleus to mitochondria. Tat-Sab ${ }_{\mathrm{KIM}}$ can specifically inhibit JNK localization to mitochondria and the activation of the mitoJNK signaling without any impact on the ratio of $\mathrm{p}-\mathrm{JNK} / \mathrm{JNK}$ or the cytosolic/nuclear JNK activation. Thus, the present study used the Tat-Sab ${ }_{\mathrm{KIM} 1}$ peptide to inhibit mitoJNK, which demonstrated a protective effect on seawater inhalation-induced ALI/ARDS. In addition, the protective effect was associated with mitoJNK. For example, Bcl-2-regulated autophagy and mitochondria-mediated apoptosis were inhibited by Tat-Sab ${ }_{\text {KIM1 }}$ pretreatment.

Several studies (31-33) have reported that JNK translocates to the nucleus after its activation and is involved in cellular functions via the phosphorylation of transcription factors in the nucleus. However, some studies $(11,34-36)$ have suggested that JNK not only serves a role in the nucleus, but also in the localization and regulation in other parts of the cells by binding to a specific protein, such as Sab (11). The carboxyl terminus of the Sab protein harbors a kinase interaction motif (KIM), which is similar to c-Jun, and JNK can bind to Sab through this motif $(11,37)$. Moreover, the carboxyl terminus of Sab also harbors four serine/-proline residues, which can also be a site that is phosphorylated by JNK (11). Therefore, Sab can not only interact with JNK but also act as the phosphorylated substrate of JNK (11). Several conformational studies $(8,11,26,34,35,37)$ have reported that Sab was localized to the mitochondria, and that a certain amount of JNK can translocate to the mitochondria by binding to the organelle. The activation of JNK and its interaction with mitochondria are involved in the regulation of mitochondrial functions $(12,34)$. The current experimental results also suggested the occurrence of mitoJNK activation in ALI, which may inhibit the energy production of mitochondria, decrease the mitochondrial membrane potential and lead to mitochondrial dysfunction. Therefore, inhibiting the translocation of JNK to the mitochondria could be used to repair damage by protecting the normal physiological function of the organelle. Previous studies $(8,34)$ have shown that inhibiting Sab expression using gene silencing technology can maintain the normal mitochondrial membrane potential and reduce the apoptosis of cells. Moreover, Chambers et al $(8,37)$ revealed that the presence of KIM1 was an essential factor for the binding and interaction between JNK and Sab. The synthesis of the KIM1-specific binding peptide Tat-Sab ${ }_{\mathrm{KIM} 1}$ may easily and effectively block the interaction between Sab and JNK, thereby inhibiting the localization of JNK to mitochondria $(6,37)$. In addition, previous studies $(6,37)$ demonstrated that Tat-Sab $\mathrm{KIM}_{\mathrm{K} 1}$ could successfully reach the cytoplasm via the cell membrane, and its concentration was stable; the concentration after $24 \mathrm{~h}$ in the cells could reach up to $90 \%$ of the initial concentration (37). Therefore, Tat-Sab ${ }_{\text {KIM1 }}$ may be used to block the binding of Sab and JNK.

There are some limitations of the present study. Firstly, as ARDS has numerous causes, further studies are required to determine whether Tat-Sab ${ }_{\mathrm{KIM} 1}$ is effective against numerous causes of ARDS. Secondly, the protective mechanism underlying blocking JNK-mitochondria interaction needs to be fully studied.

In conclusion, as anticipated, during ALI/ARDS, the activation of JNK can disrupt the normal physiological functions of the mitochondria. This disorder leads to the accumulation of ROS and increased cell apoptosis, which is a key event during ALI/ARDS. Through selective inhibition of JNK and Sab binding using Tat-Sab $\mathrm{KIM}_{\mathrm{K} 1}$, an effective and stable blocker of JNK-mitochondria interaction, the normal function of mitochondria can be maintained and the deterioration of from ALI/ARDS is blocked.

\section{Acknowledgements}

Not applicable.

\section{Funding}

This study was supported by the National Natural Science Foundation of China (grant nos. 81800076, 81900083 and 81600053).

\section{Availability of data and materials}

The datasets used and/or analyzed during the current study are available from the corresponding author on reasonable request.

\section{Authors' contributions}

CL and LB designed the study. LB and YL performed the experiments. WL and FJ performed the statistical analysis and drafted the manuscript. CL and LB confirm the authenticity of all the raw data. All authors read and approved the final manuscript.

\section{Ethics approval and consent to participate}

Not applicable.

\section{Patient consent for publication}

Not applicable.

\section{Competing interests}

The authors declare that they have no competing interests.

\section{References}

1. Musi CA, Agro G, Santarella F, Iervasi E and Borsello T: JNK3 as therapeutic target and biomarker in neurodegenerative and neurodevelopmental brain diseases. Cells-Basel 9: 2190, 2020. 
2. Tan J, Gao W, Yang W, Zeng X, Wang L and Cui X: Isoform-specific functions of c-Jun N-terminal kinase 1 and 2 in lung ischemia-reperfusion injury through the c-Jun/activator protein-1 pathway. J Thorac Cardiovasc Surg, 2020.

3. Chen M, Sun J, Lu C, Chen X, Ba H, Lin Q, Cai J and Dai J: The impact of neuronal Notch-1/JNK pathway on intracerebral hemorrhage-induced neuronal injury of rat model. Oncotarget 7 : 73903-73911, 2016.

4. Wang H, Zhong L, Mi S, Song N, Zhang W and Zhong M: Tanshinone IIA prevents platelet activation and down-regulates CD36 and MKK4/JNK2 signaling pathway. BMC Cardiovasc Disord 20: 81, 2020

5. Desai S, Laskar S and Pandey BN: Autocrine IL-8 and VEGF mediate epithelial-mesenchymal transition and invasiveness via p38/JNK-ATF-2 signalling in A549 lung cancer cells. Cell Signal 25: 1780-1791, 2013

6. Ngoei KR, Catimel B, Church N, Lio DS, Dogovski C, Perugini MA, Watt PM, Cheng HC, Ng DC and Bogoyevitch MA: Characterization of a novel JNK (c-Jun N-terminal kinase) inhibitory peptide. Biochem J 434: 399-413, 2011.

7. Matsui Y, Kuwabara T, Eguchi T, Nakajima K and Kondo M Acetylation regulates the MKK4-JNK pathway in T cell receptor signaling. Immunol Lett 194: 21-28, 2018.

8. Chambers JW, Pachori A, Howard S, Iqbal S and LoGrasso PV: Inhibition of JNK mitochondrial localization and signaling is protective against ischemia/reperfusion injury in rats. J Biol Chem 288: 4000-4011, 2013.

9. Xu J, Qin X, Cai X, Yang L, Xing Y, Li J, Zhang L, Tang Y, Liu J, Zhang $X$ and Gao F: Mitochondrial JNK activation triggers autophagy and apoptosis and aggravates myocardial injury following ischemia/reperfusion. Biochim Biophys Acta 1852: 262-270, 2015

10. Yabu T, Shiba H, Shibasaki Y, Nakanishi T, Imamura S, Touhata K and Yamashita M: Stress-induced ceramide generation and apoptosis via the phosphorylation and activation of nSMase1 by JNK signaling. Cell Death Differ 22: 258-273, 2015.

11. Wiltshire C, Matsushita M, Tsukada S, Gillespie DA and May GH A new c-Jun N-terminal kinase (JNK)-interacting protein, Sab (SH3BP5), associates with mitochondria. Biochem J 367: $577-585,2002$

12. Win S, Than TA, Le BH, Garcia-Ruiz C, Fernandez-Checa JC and Kaplowitz N: Sab (Sh3bp5) dependence of JNK mediated inhibition of mitochondrial respiration in palmitic acid induced hepatocyte lipotoxicity. J Hepatol 62: 1367-1374, 2015.

13. Li Y, Zeng Z, Li Y, Huang W, Zhou M, Zhang X and Jiang W: Angiotensin-converting enzyme inhibition attenuates lipopolysaccharide-induced lung injury by regulating the balance between angiotensin-converting enzyme and angiotensin-converting enzyme 2 and inhibiting mitogen-activated protein kinase activation. Shock 43: 395-404, 2015.

14. Hui Z, Jie H and Fan GH: Expression of DUSP12 reduces lung vascular endothelial cell damage in a murine model of lipopolysaccharide-induced acute lung injury via the apoptosis signal-Regulating Kinase 1 (ASK1)-Jun N-Terminal Kinase activation (JNK) pathway. Med Sci Monit 27: e930429, 2021.

15. Zhang ZK, Zhou Y, Cao J, Liu DY and Wan LH: Rosmarinic acid ameliorates septic-associated mortality and lung injury in mice via GRP78/IRE1alpha/JNK pathway. J Pharm Pharmacol: Mar 16, 2021 (Epub ahead of print).

16. Liu SH, Lu TH, Su CC, Lay IS, Lin HY, Fang KM, Ho TJ, Chen KL, Su YC, Chiang WC and Chen YW: Lotus leaf (Nelumbo nucifera) and its active constituents prevent inflammatory responses in macrophages via JNK/NF-kappaB signaling pathway. Am J Chin Med 42: 869-889, 2014.

17. Yu J, Wang Y, Li Z, Dong S, Wang D, Gong L, Shi J, Zhang Y, Liu D and Mu R: Effect of Heme Oxygenase-1 on Mitofusin-1 protein in LPS-induced ALI/ARDS in rats. Sci Rep 6: 36530 , 2016.

18. Suresh K, Servinsky L, Reyes J, Baksh S, Undem C, Caterina M, Pearse DB and Shimoda LA: Hydrogen peroxide-induced calcium influx in lung microvascular endothelial cells involves TRPV4. Am J Physiol Lung Cell Mol Physiol 309: L1467-L1477, 2015.

19. Wang K, Chen Y, Zhang P, Lin P, Xie N and Wu M: Protective features of autophagy in pulmonary infection and inflammatory diseases. Cells Basel 8: 123, 2019.
20. Jackson MV,MorrisonTJ,DohertyDF,McAuley DF,Matthay MA, Kissenpfennig A, O'Kane CM and Krasnodembskaya AD: Mitochondrial transfer via tunneling nanotubes is an important mechanism by which mesenchymal stem cells enhance macrophage phagocytosis in the in vitro and in vivo models of ARDS Stem Cells 34: 2210-2223, 2016.

21. Islam MN, Das SR, Emin MT, Wei M, Sun L, Westphalen K, Rowlands DJ, Quadri SK, Bhattacharya S and Bhattacharya J: Mitochondrial transfer from bone-marrow-derived stromal cells to pulmonary alveoli protects against acute lung injury. Nat Med 18: 759-765, 2012

22. Gomzikova MO, James V and Rizvanov AA: Mitochondria donation by mesenchymal stem cells: Current understanding and mitochondria transplantation strategies. Front Cell Dev Biol 9: 653322, 2021.

23. Zhang M, Gao Y, Zhao W, Yu G and Jin F: ACE-2/ANG1-7 ameliorates ER stress-induced apoptosis in seawater aspiration-induced acute lung injury. Am J Physiol Lung Cell Mol Physiol 315: L1015-L1027, 2018.

24. National Research Council (US) Committee for the Update of the Guide for the Care and Use of Laboratory Animals: Guide for the Care and Use of Laboratory Animals. 8th edition. National Academies Press (US), Washington, DC, 2011.

25. Li C, Liu M, Bo L, Liu W, Liu Q, Chen X, Xu D, Li Z and Jin F: NFAT5 participates in seawater inhalationinduced acute lung injury via modulation of NF-kappaB activity. Mol Med Rep 14: 5033-5040, 2016.

26. Hanawa N, Shinohara M, Saberi B, Gaarde WA, Han D and Kaplowitz N: Role of JNK translocation to mitochondria leading to inhibition of mitochondria bioenergetics in acetaminophen-induced liver injury. J Biol Chem 283: 13565-13577, 2008.

27. Li X, Wang Y, Xiong Y, Wu J, Ding H, Chen X, Lan L and Zhang H: Galangin induces autophagy via deacetylation of LC3 by SIRT1 in HepG2 cells. Sci Rep 6: 30496, 2016.

28. Xu HD and Qin ZH: Beclin 1, Bcl-2 and autophagy. Adv Exp Med Biol 1206: 109-126, 2019.

29. Rubenfeld GD, Caldwell E, Peabody E, Weaver J, Martin DP, Neff M, Stern EJ and Hudson LD: Incidence and outcomes of acute lung injury. N Engl J Med 353: 1685-1693, 2005.

30. Fan E, Brodie D and Slutsky AS: Acute respiratory distress syndrome: Advances in diagnosis and treatment. JAMA 319: 698-710, 2018.

31. FarkhondehT,MehrpourO,Buhrmann C,Pourbagher-ShahriAM, Shakibaei $M$ and Samarghandian S: Organophosphorus compounds and MAPK signaling pathways. Int J Mol Sci 21: 4258,2020

32. Han X, Liu C, Zhang K, Guo M, Shen Z, Liu Y, Zuo Z, Cao M and Li Y: Calpain and JNK pathways participate in isofluraneinduced nucleus translocation of apoptosis-inducing factor in the brain of neonatal rats. Toxicol Lett 285: 60-73, 2018

33. Li Y, Xu M, Ding X, Yan C, Song Z, Chen L, Jian Y, Tang G, Tang C, Di Y, et al: Protein kinase C controls lysosome biogenesis independently of mTORC1. Nat Cell Biol 18: 1065-1077, 2016.

34. Win S, Than TA, Fernandez-Checa JC and Kaplowitz N: JNK interaction with Sab mediates ER stress induced inhibition of mitochondrial respiration and cell death. Cell Death Dis 5: e989, 2014.

35. Chambers JW, Howard S and LoGrasso PV: Blocking c-Jun $\mathrm{N}$-terminal kinase (JNK) translocation to the mitochondria prevents 6-hydroxydopamine-induced toxicity in vitro and in vivo. J Biol Chem 288: 1079-1087, 2013.

36. Zhao Y and Herdegen T: Cerebral ischemia provokes a profound exchange of activated JNK isoforms in brain mitochondria. Mol Cell Neurosci 41: 186-195, 2009.

37. Chambers JW, Cherry L, Laughlin JD, Figuera-Losada M and Lograsso PV: Selective inhibition of mitochondrial JNK signaling achieved using peptide mimicry of the Sab kinase interacting motif-1 (KIM1). ACS Chem Biol 6: 808-818, 2011.

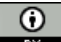

This work is licensed under a Creative Commons Attribution 4.0 International (CC BY 4.0) License. 